
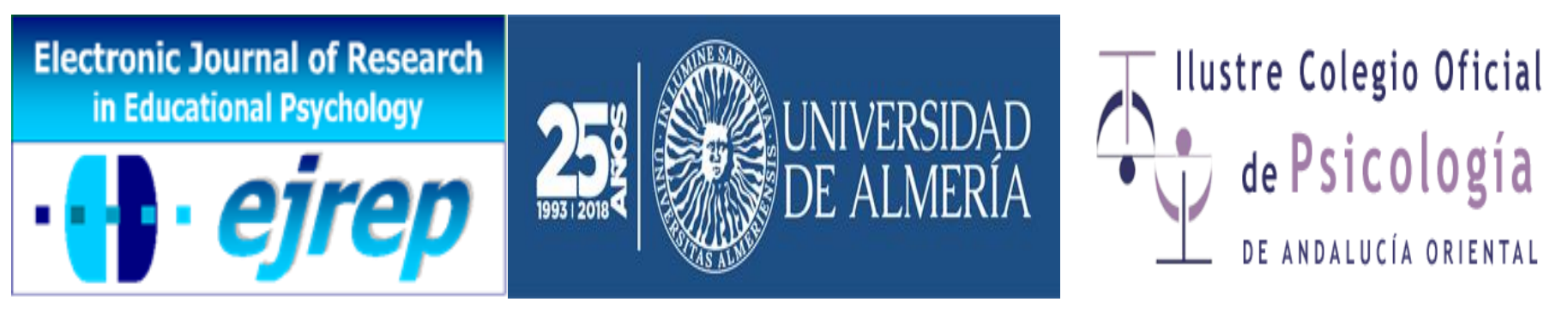

\title{
Niños con altas capacidades: Análisis de las variables familiares implicadas en el desarrollo del potencial
}

\section{Blanca Ivet Chávez Soto, Guadalupe Acle Tomasini}

Facultad de Psicología, Residencia en Educación Especial, Facultad de Estudios

Superiores Zaragoza

Universidad Nacional Autónoma de México

México

Correspondencia: Blanca Ivet Chávez Soto: $2^{a}$ avenida, No 73, Col. El Sol, Cd Nezahualcóyotl, Estado de México, México E-mail: mil_chavez@hotmail.com

(C) Universidad de Almería and Ilustre Colegio Oficial de la Psicología de Andalucía Oriental (Spain) 


\section{Resumen}

Introducción. Existen diferentes factores familiares que influyen en la transformación del potencial de los alumnos con altas capacidades, los estudios han encontrado que la ocupación de los padres, el tiempo que se dedica a los niños, las prácticas de crianza y las expectativas son aspectos que intervienen en el desarrollo de las habilidades. El objetivo del trabajo fue reconocer las variables que caracterizan a los padres de alumnos con altas capacidades de educación primaria de una zona urbano marginal.

Método. Se trabajó en tres escuelas primarias públicas ubicadas al oriente de la Ciudad de México. Participaron 28 niños con altas capacidades, sus padres y 24 profesores. A los alumnos se les aplicaron pruebas para evaluar su inteligencia, creatividad, compromiso con la tarea y el autoconcepto académico. Los maestros contestaron una lista de nominación y los padres respondieron una lista de cotejo para reconocer las características de las familias.

Resultados. Se observó que existe un $4 \%$ de estudiantes con altas capacidades que viven en zonas vulnerables. Con la correlación de Spearman Rho, se observó que independientemente del nivel socioeconómico de los padres, ellos tratan de proveerles actividades adicionales a sus hijos para favorecer sus capacidades. La escolaridad de las madres ayuda a que busquen alternativas adicionales y de bajo costo. Asimismo, el tiempo que ellas dedican para hacer la tarea afecta de forma negativa al autoconcepto académico de los niños.

Discusión y conclusión. Se logró reconocer la influencia de algunas variables familiares que favorecen el potencial de los estudiantes con altas capacidades. Es necesario continuar con este tipo de investigaciones para reconocer cuáles son las características de los padres y proveerles apoyos adicionales para lograr favorecer un óptimo desarrollo de los niños.

Palabras Clave: inteligencia, creatividad, características de los padres, autoconcepto académico, compromiso con la tarea 


\begin{abstract}
Introduction. There are different family factors that influence the transformation of the potential of students with high abilities, studies have found that the occupation of parents, the time spent on children, parenting practices and expectations are aspects that influence the development of skills. The objective of the work was to recognize the variables that characterize the parents of students with high primary education capacities in a marginal urban area.

Method. It worked in three public elementary schools located east of Mexico City, participated 28 children with high abilities, their parents and 24 teachers. Tests were applied to evaluate the intelligence, creativity, task commitment and academic self-concept of the students. The teachers answered a nomination list and the parents responded to a checklist to recognize the characteristics of the families.
\end{abstract}

Results. It was observed that there is a $4 \%$ of students with high abilities whom live in vulnerable areas. With the Spearman Rho correlation, it was observed that regardless of the socioeconomic level of the parents, they try to provide additional activities to their children to favor their abilities. The mothers' schooling helps them look for additional and low-cost alternatives. Also, the time they spend doing homework, affects in a negatively way the academic self-concept of children.

Discussion and conclusion. It was possible to recognize the influence of some family variables that help the development of the potential of students with high abilities. It is necessary to continue with this type of research in order to recognize the characteristics of the parents and provide them with additional supports and strategies to achieve an optimal development of the children.

Keywords: Intelligence, creativity, characteristics of parents, academic self-concept, commitment to homework 


\section{Introducción}

Durante años los investigadores se han interesado por el estudio de los alumnos con altas capacidades. Tal es el caso de Galton, quien mencionó que la herencia era el principal factor para explicar los elevados niveles intelectuales. Otros destacaron la influencia de las variables personales como la motivación, autoconcepto, persistencia, autocontrol y liderazgo que se vinculan con el desarrollo de las habilidades (Mönks y Van Boxtel, 1992; Renzulli, 2011). Las aproximaciones más resientes han reconocido la relevancia del contexto familiar y escolar para favorecer las capacidades cognoscitivas de los niños (Gagné, 2012; Piirto y Fraas, 2012). Para Castellanos, Bazán, Ferrari y Hernández (2015) existen diferentes factores familiares que influyen en la transformación del potencial de los alumnos. Por lo que han considerado que la presencia de distintos talentos se debe a la configuración dinámica de un conjunto heterogéneo de procesos, cualidades y formaciones psicológicas en interacción con las variables de su contexto. Aun cuando existe una gran diversidad en las familias, los datos han revelado algunos aspectos importantes que promueven las altas capacidades como la ocupación de los padres, el tiempo que se dedica a los niños, la planificación e instrucción, las prácticas de crianza y las expectativas sirven de modelo para el éxito académico.

En relación con los estudiantes que presentan altas capacidades se ha observado que la familia juega un rol trascendente en el desarrollo de sus habilidades por lo que es necesario adoptar un enfoque ecológico para la identificación de dicha población (Mönks y Van Boxtel, 1992). Los padres constituyen una fuente valiosa de información, por lo general sus observaciones son objetivas y precisas, especialmente en los procesos evolutivos. Al convivir con sus hijos en distintas situaciones, pueden apreciar conductas que no se ven en el contexto escolar, conocen sus gustos e intereses, pero en ocasiones desconocen que tienen altas capacidades o por el contrario creen que son talentosos sin que realmente lo sean (Blanco, 2001). Autores como Gómez y Valadez (2010) Martín (2004) y Olszewski-Kubilius (2008) indicaron que los papás de niños con altas capacidades en ocasiones experimentan tensión ante el aumento en las demandas que significa ser un alumno con estas características, por lo que es frecuente encontrar que están abrumados por la inquietud, impaciencia y preguntas de sus hijos lo que puede generar un 
deterioro en la relación con ellos, también es posible que los progenitores fomenten la ansiedad de sus niños si tienen expectativas muy elevadas en cuanto a su rendimiento escolar real.

Lo anterior, refleja que el ambiente familiar es de suma importancia para la identificación y el desarrollo de las habilidades de los estudiantes con altas capacidades. Se sabe que los padres proveen de información que ayuda a conocer los talentos e intereses de sus hijos; pero también necesitan propuestas para brindar alternativas educativas que permitan enriquecer el entorno de los niños. Al respecto, Gagné (2012), Mönks y Van Boxtel (1992), Piirto (1999) han destacado que es necesario considerar una visión multidimensional para explicar las características de los alumnos con altas capacidades ya que al igual que cualquier otro individuo, se desarrollan e interaccionan en marcos sociales y experimentan procesos evolutivos complejos que deben ser estudiados. De esta manera, el presente trabajo se fundamenta en el modelo teórico de la interdependencia triádica diseñado por Mönks y Van Boxtel (1992) quienes ampliaron la visión propuesta por Renzulli (1986) al mencionar que los alumnos con aptitud sobresaliente son aquellos que demuestran una capacidad cognitiva superior, altos niveles de creatividad y motivación, además se estableció como otro elemento importante al autoconcepto académico y tres marcos sociales específicos: la escuela, los compañeros y la familia.

Para la identificación de esta población se han considerado principalmente las pruebas para evaluar la inteligencia, otras propuestas han indicado que existen distintas variables que influyen en la presencia del potencial como lo es la creatividad, el autoconcepto académico y el compromiso con la tarea (Bernal, Esparza, Ruiz, Ferrando \& Sainz, 2017; Espinoza \& Reyes, 2008; Ferrando, Prieto, Ferrándiz \& Sánchez, 2005; Manzano \& Arranz, 2008; Preckel, Goetz, Pekrun \& Kleine, 2008; Rudasill, Capper, Foust, Callahan \& Albaugh, 2009; Renzulli, 1986; Schick \& Phillipson, 2009). A partir de estas alternativas y con el propósito de incluir otros factores que actúan como moderadores se ha incorporado la evaluación de algunos aspectos de la familia debido a que el desarrollo de las altas capacidades depende esencialmente del ambiente social, el apoyo, la comprensión y estimulación adecuada de padres (Borges, Hernández \& Rodríguez, 2006; Chan, 2005; Garn, Matthews \& Jolly, 2010; Gómez \& Valadez, 2010; Manzano \& Arranz, 2008; Mönks \& Van Boxtel, 1992; Piirto, 1999). 
Lo anterior cobra particular importancia cuando se trabaja en comunidades urbano marginales, con alta vulnerabilidad social y económica ya que, como lo reportaron los estudios de Acle y Ordaz (2010), Burney y Beike (2008), Ocampo y López (2011), Pérez y González (2007), Sarouphim (2008), Valadez, Pérez y Beltrán (2010) existen alumnos con altas capacidades a los cuales no se les identifica debido a que existen variables asociadas con las condiciones de pobreza que afectan sus experiencias educativas, lo que contribuye a las bajas puntuaciones en las pruebas de inteligencia y al deterioro de sus talentos por lo que se encuentran en situación de riesgo educativo y social. Por lo que se sugiere realizar la identificación a partir de modelos multidimensionales en los que se incluyan diversos factores personales y familiares, principalmente en aquellos grupos que se encuentran en zonas vulnerables o de riesgo.

\section{Objetivo e hipótesis}

A partir de lo anterior, el objetivo del trabajo fue reconocer las variables que caracterizan a los padres de alumnos con altas capacidades de educación primaria de una zona urbano marginal. De acuerdo con la evidencia empírica (Borges, Hernández y Rodríguez, 2006; Chan, 2005; Garn, Matthews, Jolly, 2010; Gómez y Valadez, 2010; Manzano y Arranz, 2008) se reconoce que existen algunas características de la familia (estructura, nivel socioeconómico, religión, apoyo familiar) en específico de los padres (edad, tiempo de dedicación a los hijos, escolaridad, ocupación) que actúan como moderadores de las altas capacidades de los estudiantes.

\section{Método}

\section{Participantes}

Se empleó un muestreo no probabilístico intencional. Se identificaron a 28 niños (18 mujeres y 10 hombres) que presentaron altas capacidades, con un rango de edad de ocho a 11 años $\left(M_{\text {edad }}=9.94, D E=0.840\right)$, dos estudiantes de tercero, 10 de cuarto y 16 de quinto grado de primaria. También participaron los padres de familia de estos estudiantes. En cuanto a los datos de las madres se encontró que tenían un rango de edad de 24 a 49 años $\left(M_{\text {edad }}=37.96, D E=\right.$ 6.028). En el caso de los padres su edad estuvo comprendida entre los 32 y 54 años (Medad $=$ 
42.36, $D E=6.170$ ). Además, colaboraron 24 profesores, por cada grado escolar había ocho profesores, en promedio tenían 18 años de experiencia docente.

\section{Instrumentos}

Test de Matrices Progresivas Raven Forma Coloreada (Raven, Court \& Raven, 1993): Mide la capacidad intelectual del niño a través del factor "g"; consta de 36 problemas, distribuidos en tres series A-AB-B, los cuales están ordenados de menor a mayor dificultad, la respuesta correcta de cada ejercicio se encuentra mezclada entre otras cinco erróneas. Se realizó una confiabilidad mediante un test- retest y se obtuvo una $r=0.774$, también se hizo un Alpha de Cronbach de 0.88, ambos resultados apoyan la consistencia del instrumento (Chávez, 2014).

Prueba de Pensamiento Creativo Versión Figural A (Torrance, 2008): Su propósito es evaluar las producciones creativas de las personas con tres actividades que se califican con los indicadores de fluidez, originalidad, elaboración, títulos y cierre. Se obtuvo la validez de constructo y mostró un índice de confiabilidad de 0.90 obtenido por el Alfa de Cronbach (Zacatelco, Chávez, González \& Acle, 2013).

Escala de Compromiso con la Tarea (Zacatelco, 2005): Evalúa los niveles de la motivación intrínseca en áreas curriculares y extracurriculares a través de los indicadores: interés, persistencia y esfuerzo. Consta de 18 reactivos tipo likert, con seis intervalos de respuesta. El instrumento fue validado en tres escuelas de la delegación Iztapalapa con una confiabilidad de 0.79 obtenida por un Alfa de Cronbach.

Prueba de Autoconcepto Académico (Chávez, 2014): Consta de 36 ítems con formato tipo likert, ayuda a medir la percepción que tiene el alumno sobre su rendimiento en las materias escolares: matemáticas, español, historia, ciencias naturales, educación física y artística. El instrumento obtuvo una confiabilidad de 0.848 a través de un Alfa de Cronbach. 
Nominación de Maestros para Identificar Potencial Sobresaliente (Zacatelco, Chávez \& González, 2013): Tiene como objetivo conocer la percepción de los maestros a partir de los factores propuestos por Renzulli (compromiso con la tarea, capacidad superior y creatividad) además se agregó una cuarta dimensión que corresponde a los aspectos socioafectivos. Consta de dos partes en la primera están los datos del profesor y del alumno, en la segunda hay 37 ítems dicotómicos. La confiabilidad total de la prueba fue de 0.934 obtenida por un Alfa de Cronbach.

Lista de chequeo para padres de familia (Chávez, 2014): Desarrollada para esta investigación con el propósito de conocer aspectos sociodemográficos de los padres de familia como: edad, escolaridad, ocupación, religión, estado civil, estructura familiar, tiempo que dedican al niño en sus tareas, algunos aspectos vinculados con sus hijos y el nivel socioeconómico, este último dato se obtuvo a través de la clasificación propuesta por la Asociación Mexicana de Agencias de Mercados y Opinión Pública -AMAI- (2017) en el que se consideran seis niveles:

- A/B clase alta: es el segmento con más alto nivel de vida, el perfil del jefe de familia está conformado por personas con escolaridad de licenciatura o posgrado, viviendas propias con lujos, todos los servicios y comodidades.

- C+ clase media alta: Incluye a personas cuyos ingresos y/o estilo de vida es ligeramente superior a los de clase media; el perfil del jefe de familia de estos hogares está formado por individuos con licenciatura completa, con viviendas propias que incluyen algunos lujos y cuentan con todas las comodidades.

- C clase media: En este rubro están las personas que tiene como escolaridad preparatoria, tienen viviendas propias y con algunas comodidades.

- D+ clase media baja: Se incluyen hogares con ingresos y/o estilos de vida ligeramente menores a los de la clase media; son quienes llevan un mejor estilo dentro de la clase baja, el perfil del jefe de familia es con escolaridad de carreras técnica, secundaria o primaria completa. Poseen casa propia, inmuebles rentados o de interés social.

- D clase baja: aquí se ubican los individuos con nivel educativo de primaria, quienes habitan en vecindades y unidades de interés social, propias o rentadas. 
- E clase más baja: El jefe de familia usualmente tiene la primaria incompleta, en ocasiones las personas de este segmento suelen carecer de propiedades, por lo que rentan viviendas generalmente austeras y en el mismo lugar habitan más de una generación.

\section{Procedimiento}

Para reconocer a los alumnos con altas capacidades se realizó lo siguiente: Se solicitó permiso a los directores y maestros de las tres escuelas primarias. Los padres de familia firmaron el consentimiento informado y se contó con el asentimiento de los niños. La población inicial fue de 670 niños (356 mujeres y 314 hombres) con un promedio de edad de $9.18(D E=0.997)$ que cursaban de tercero a quinto grado. La aplicación de los instrumentos se realizó durante cuatro semanas para cada colegio, el procedimiento general fue el que se describe a continuación:

1) Se platicó con los docentes para que contestar la Lista de Nominación del Maestro, después los niños realizaron la Prueba de Pensamiento Creativo.

2) Durante la segunda semana se contestó la Prueba de Compromiso con la Tarea.

3) En la tercera se resolvió el Test de Matrices Progresiva Raven.

4) En la cuarta los alumnos contestaron la Prueba de Autoconcepto Académico.

Se diseñó una base de datos en el programa estadístico SPSS versión 20, se capturaron los nombres, edades, sexo y las puntuaciones obtenidas en los instrumentos por cada uno de los alumnos. Se encontraron los valores mínimos, máximos, medias, la desviación estándar y el percentil 75. Cabe indicar que, para la identificación de los alumnos con altas capacidades se tomaron en cuenta aquellos niños que obtuvieron percentiles iguales o por encima del centil 75 en tres de las cinco pruebas aplicadas ya que de acuerdo con diversos autores es la puntuación que indica un claro perfil sobresaliente (Mönks \& Van Boxtel, 1992; Renzulli, 2011; Prieto, 1999; Zacatelco, 2005). Posteriormente, los niños detectados se organizaron en tres categorías de acuerdo con la cantidad de variables en las que obtuvieron percentiles iguales o superiores al 75:

- Grupo 1: Alumnos que presentaron altas puntuaciones en los cinco instrumentos.

- Grupo 2: Aquellos que obtuvieron elevadas estimaciones en cuatro variables.

- Grupo 3: Niños con puntajes superiores o iguales al centil 75 en tres de las pruebas. 
Se elaboró una lista de chequeo para padres de familia con el propósito de conocer sus características, para su diseño se consideraron algunas dimensiones que han sido reportadas en distintos estudios desarrollados con los alumnos que presentan altas capacidades (Borges et al., 2006; Chamberlin \& Chamberlin, 2010; Elices, Palazuelo \& Del Caño, 2006; Gómez \& Valadez, 2010; Hernández \& Borges, 2010; Manzano \& Arranz, 2008; Morawska \& Sanders, 2009; Zeynep \& Bayindir, 2009). Después de la identificación de los estudiantes se contactó a los padres de familia, para que asistieran a una junta y se solicitó su ayuda para contestar la lista de chequeo.

\section{Análisis de datos}

Con la información recabada se elaboró una base de datos en el programa estadístico SPSS versión 20, en la que se incluyeron las puntuaciones de los instrumentos de los niños con altas capacidades, así como los datos proporcionados por los padres en las listas de chequeo. Posteriormente, se realizaron los siguientes análisis: la estadística descriptiva para conocer las características de la muestra, la prueba no paramétrica Kruskal- Wallis para determinar diferencias por edad de los padres entre los tres grupos de estudiantes con altas capacidades, se empleó una correlación de Spearman Rho para conocer la asociación entre las variables del estudio.

\section{Resultados}

Para identificar y reconocer las características de los niños con altas capacidades, se obtuvieron las puntuaciones mínimas, máximas, media, desviación estándar y percentil 75 de los instrumentos empleados: Test de Matrices Progresivas, Prueba de Pensamiento Creativo, Prueba de Compromiso con la Tarea, Prueba de Autoconcepto Académico y Lista de Nominación del Maestro (Tabla 1). 
Tabla 1. Puntuaciones de las pruebas empleadas en la identificación de alumnos con altas capacidades

\begin{tabular}{lcccccc}
\hline \multicolumn{1}{c}{ INSTRUMENTOS } & \multicolumn{2}{c}{ Puntuaciones } & \multirow{2}{*}{ MEDIA } & \multirow{2}{*}{ D.E } & \multicolumn{2}{c}{ PERCENTIL } \\
\cline { 2 - 5 } & Mínimas & Máximas & & $\mathbf{7 5}$ \\
\hline Test de Matrices Progresivas Raven & 2 & 36 & 26.32 & 5.8 & $\mathbf{3 1}$ \\
Prueba de Pensamiento Creativo & 7 & 94 & 50.86 & 15.05 & $\mathbf{6 1}$ \\
Prueba de Compromiso con la Tarea & 28 & 106 & 77.12 & 15.33 & $\mathbf{8 9}$ & $\mathbf{1 7 5}$ \\
Prueba de Autoconcepto Académico & 55 & 217 & 159.92 & 22.63 & $\mathbf{2 6}$ \\
Lista de Nominación del Maestro & 0 & 37 & 16.13 & 11.33 & \\
\hline
\end{tabular}

El análisis de los datos permitió observar que, de los 670 alumnos de la muestra, 28 se ubicaron con altas capacidades, lo que corresponde al $4 \%$ de la población. Cabe indicar que, con estos estudiantes se conformaron tres grupos de acuerdo con el número de variables en las que obtuvieron puntuaciones iguales o superiores al percentil 75. En este sentido, el grupo I se conformó por diez niños que presentaron estimaciones altas en las cinco pruebas, en el grupo II se ubicaron a nueve estudiantes con valores por encima del centil 75 en cuatro dimensiones y el grupo III se constituyó con nueve estudiantes quienes obtuvieron puntajes elevados en por lo menos tres de los instrumentos empleados.

Características que los padres indicaron con respecto a sus hijos con altas capacidades

En la Tabla 2 se presenta la información proporcionada por los padres relacionada con características de sus hijos. En esta muestra se encontró que el grupo I, estaba conformado por siete niños primogénitos y tres nacieron en segundo lugar. En el grupo II, se reportó que tres eran primogénitos, uno ocupaba el segundo lugar, cuatro el tercero y uno el cuarto. En cuanto al grupo III, se observó que dos eran primogénitos, dos en el segundo, dos en el tercer lugar y uno en el cuarto. Estos datos son interesantes y coinciden con lo encontrado por Kristensen y Bjerkedal (2007), Ordaz y Acle (2013) Zacatelco y Acle (2009) quienes reportaron que era más común encontrar características de altas capacidades en hijos primogénitos.

Por otro lado, en una de las preguntas se indagó sobre cómo eran emocionalmente sus hijos. Los datos revelaron que, en el primer grupo, siete de los padres los consideraron sensibles y tres amables. En el segundo, cinco niños fueron caracterizados como sensibles, tres tímidos y uno hiperactivo. Con respecto al grupo III, los padres indicaron que siete eran sensibles y dos amables (Tabla 2). En este sentido, autores como Piechowsky y Colageno (1984) han Electronic Journal of Research in Educational Psychology, 16(2), 273-300. ISSN: 1696-2095. 2018. no. 45 
profundizado sobre la sensibilidad de estos estudiantes y señalaron que este factor contribuye a la forma en como manejan y experimentan la intensidad y complejidad de los sentimientos.

Tabla 2. Características de los niños sobresalientes por grupo

\begin{tabular}{|c|c|c|c|}
\hline & GRUPO I & GRUPO II & GRUPO III \\
\hline $\begin{array}{l}\text { Lugar que ocupa entre } \\
\text { hermanos }\end{array}$ & $\begin{array}{l}\text { - Siete primogénitos } \\
\text { - Tres en segundo }\end{array}$ & $\begin{array}{l}\text { - Tres primogénitos } \\
\text { - Uno en segundo } \\
\text { - Cuatro en tercero } \\
\text { - Uno cuarto }\end{array}$ & $\begin{array}{l}\text { - Dos primogénitos } \\
\text { - Dos en segundo } \\
\text { - Cuatro en tercero } \\
\text { - Uno en cuarto }\end{array}$ \\
\hline $\begin{array}{l}\text { Como consideran que son } \\
\text { sus hijos }\end{array}$ & $\begin{array}{l}\text { - Siete sensibles } \\
\text { - Tres amables }\end{array}$ & $\begin{array}{l}\text { - Cinco sensibles } \\
\text { - Tres tímidos } \\
\text { - Uno hiperactivo }\end{array}$ & $\begin{array}{l}\text { - Siete sensibles } \\
\text { - Dos amables }\end{array}$ \\
\hline
\end{tabular}

\section{Características de las madres}

Los resultados de las características evaluadas en las madres de acuerdo con el grupo de alta capacidad de sus hijos se muestran en la Tabla 3. Con respecto a la variable edad, se observó que las mamás del grupo I tenían una edad media menor en comparación con las de los otros dos grupos, la prueba no paramétrica Kruskal Wallis mostró que no existían diferencias significativas $\left(F_{(2,32)}=0.313, p<0.05\right)$. En cuanto a la ocupación de las madres, se encontró que en el grupo I, ocho se dedicaban al hogar y dos eran profesionistas. En el segundo grupo, seis se desempeñaban como amas de casa, una era comerciante y otra profesionista. En el grupo III, cuatro se ocupaban del hogar, tres mencionaron ser comerciantes, una secretaria y una empleada doméstica. En la escolaridad, se encontró que en el grupo I, tres concluyeron la secundaria, tres la preparatoria, una tenía carrera técnica y tres terminaron la licenciatura. En el grupo II, cinco tenían secundaria, una preparatoria, otra carrera técnica y dos con licenciatura. En el grupo III, había tres con secundaria, tres con preparatoria, una concluyó carrera técnica y dos la licenciatura (Tabla 3). 
Tabla 3. Característica de las madres por grupo de niños con altas capacidades

\begin{tabular}{|c|c|c|c|}
\hline & \multicolumn{3}{|c|}{ GRUPO EN EL CUAL SE UBICÓ A LAS MADRES } \\
\hline & GRUPO I & GRUPO II & GRUPO III \\
\hline Edad promedio & $-\quad M=35.6$ & $\mathrm{M}=37.67$ & $\mathrm{M}=40.67$ \\
\hline Escolaridad & $\begin{array}{ll}\text { - } & \text { Tres con secundaria } \\
\text { - } & \text { Tres con preparatoria } \\
\text { - } & \text { Una carrera técnica } \\
\text { - } & \text { Tres con licenciatura }\end{array}$ & $\begin{array}{ll}\text { - } & \text { Cinco con secundaria } \\
\text { - } & \text { Una con preparatoria } \\
\text { - } & \text { Una con carrera técnica } \\
\text { - } & \text { Dos con licenciatura }\end{array}$ & $\begin{array}{ll}- & \text { Tres con secundaria } \\
\text { - } & \text { Tres con preparatoria } \\
\text { - } & \text { Una con carrera técnica } \\
\text { - } & \text { Dos con licenciatura }\end{array}$ \\
\hline Ocupación & $\begin{array}{ll}\text { - } & \text { Ocho al hogar } \\
\text { - } & \text { Dos profesionistas }\end{array}$ & $\begin{array}{ll}\text { - } & \text { Seis al hogar } \\
\text { - } & \text { Una comerciante } \\
\text { - } & \text { Una profesionista }\end{array}$ & $\begin{array}{ll} & \text { Cuatro al hogar } \\
\text { - } & \text { Tres comerciantes } \\
\text { - } & \text { Una secretaria } \\
\text { - } & \text { Una empleada doméstica }\end{array}$ \\
\hline $\begin{array}{l}\text { Tiempo que } \\
\text { dedican para } \\
\text { hacer la tarea }\end{array}$ & $\begin{array}{ll}\text { - } & \text { Nueve de } 1 \text { a } 5 \text { horas } \\
\text { - } & \text { Una no lo hacen }\end{array}$ & $\begin{array}{ll}\text { - } & \text { Ocho de } 1 \text { a } 5 \text { horas } \\
\text { - } & \text { Una no lo hace }\end{array}$ & $\begin{array}{ll}\text { - } & \text { Cuatro de } 1 \text { a } 5 \text { horas } \\
\text { - } & \text { Cuatro de } 6 \text { a } 10 \text { horas } \\
\text { - } & \text { Una no lo hace }\end{array}$ \\
\hline
\end{tabular}

Con relación al tiempo que dedicaban las madres para hacer la tarea con sus hijos, se observó que nueve mamás del grupo I y ocho del grupo II, empleaban entre una y cinco horas para realizar las actividades escolares, en comparación con las del grupo III, en donde sólo cuatro mamás reportaron esa misma cantidad de tiempo (Tabla 3). Estos datos resultaron interesantes pues mostraron que aun cuando las madres de los niños con altas capacidades no cuentan con elevados niveles educativos ni ocupacionales, ellas les brindan el tiempo requerido para ayudarles de forma adecuada en sus estudios (Borges et al., 2006).

\section{Características de los padres}

En la Tabla 4 se presentan las características de los padres de acuerdo con el grupo de alta capacidad en el cual se ubicó su hijo. Se encontró que en el caso de la edad las puntuaciones medias de los papás de los tres grupos fueron similares, la prueba no paramétrica Kruskal Wallis mostró que no existían diferencias significativas $\left[F_{(2,32)}=0.930, p<0.05\right]$. En la ocupación de los padres, se observó que en el grupo I, hay dos docentes, un profesionista, un chofer, un impresor, un empleado, un obrero, un oficinista, un transportista y un desempleado. En el segundo grupo, se encontraron dos empleados, un comerciante, un profesionista, un albañil y un mecánico; en el tercero, había tres comerciantes, cuatro empleados y un obrero. El nivel educativo de los padres fue el siguiente: en el primer grupo, uno terminó la primaria, uno concluyó la secundaría, dos la preparatoria, cuatro la licenciatura y dos la maestría. En el segundo 
grupo, dos papás tenían secundaría, uno preparatoria, otro con carrera técnica y tres con licenciatura. Los datos reportados por los padres del grupo III, indicaron que uno tenía primaria, dos con secundaria, dos tenían preparatoria, dos con carrera técnica y uno licenciatura (Tabla 4).

Tabla 4. Característica de los padres por grupo de niños con altas capacidades

\begin{tabular}{|c|c|c|c|}
\hline & \multicolumn{3}{|c|}{ GRUPO EN EL CUAL SE UBICÓ A LOS PADRES } \\
\hline & GRUPO I & GRUPO II & GRUPO III \\
\hline Edad promedio & $-\quad M=42$ & - $\quad M=42$ & - $M=43.13$ \\
\hline Escolaridad & $\begin{array}{ll}\text { - } & \text { Uno con primaria } \\
\text { - } & \text { Uno con secundaria } \\
\text { - } & \text { Dos con preparatoria } \\
\text { - } & \text { Cuatro con } \\
& \text { licenciatura } \\
\text { - } & \text { Dos con maestría } \\
\end{array}$ & $\begin{array}{l}\text { - Dos con secundaria } \\
\text { - Uno con preparatoria } \\
\text { - Uno con carrera } \\
\text { técnica } \\
\text { - Tres con licenciatura }\end{array}$ & $\begin{array}{l}\text { - } \text { Uno con primaria } \\
\text { - } \text { Dos con secundaria } \\
\text { - } \text { Dos con preparatoria } \\
\text { - } \text { Dos con carrera técnica } \\
\text { - }\end{array}$ \\
\hline Ocupación & $\begin{array}{l}\text { - Un chofer } \\
\text { - Un profesionista } \\
\text { - } \text { - Dos docentes } \\
\text { - Un impresor } \\
\text { - Un transportista } \\
\text { - } \\
\text { - Un empleado } \\
\text { - Un obrero } \\
\text { - Un oficinista } \\
\end{array}$ & $\begin{array}{l}\text { - Un comerciante } \\
\text { - Dos empleados } \\
\text { - Un profesionista } \\
\text { - Un albañil } \\
\text { - Un mecánico }\end{array}$ & $\begin{array}{l}\text { - } \text { Tres comerciantes } \\
\text { - } \text { Cuatro empleados } \\
\text { - } \text { Un obrero }\end{array}$ \\
\hline $\begin{array}{l}\text { Tiempo que dedican } \\
\text { para hacer la tarea }\end{array}$ & $\begin{array}{ll}\text { - } & \text { Siete de } 1 \text { a } 5 \text { horas } \\
\text { - } & \text { Tres no lo hacen }\end{array}$ & $\begin{array}{ll}\text { - } & \text { Tres de } 1 \text { a } 5 \text { horas } \\
\text { - } & \text { Cuatro no lo hacen }\end{array}$ & $\begin{array}{l}\text { - } \text { Cinco de } 1 \text { a } 5 \text { horas } \\
\text { - Dos no lo hacen }\end{array}$ \\
\hline
\end{tabular}

Con respecto a los padres, en el grupo I se encontró que siete de ellos dedicaban de una a cinco horas al día para hacer la tarea con sus hijos y tres no realizaban dicha actividad. Para el segundo grupo, sólo tres de los padres empleaban de una a cinco horas y cuatro no participaban en las tareas escolares de sus hijos. En el tercero, se reportó que cinco padres utilizaban de una a cinco horas al día y que dos, no llevaban a cabo esta labor con sus hijos (Tabla 4). En este sentido, cobra particular importancia lo encontrado por Pougnet, Servin, Statk y Schwartzman (2011) quienes indicaron que la figura paterna en la infancia promueve el comportamiento afectivo e intelectual de los niños.

\section{Características de la Familia}

Las variables familiares que se investigaron en este trabajo se muestran en la Tabla 5. Se encontró que en el grupo I, siete de los padres eran casados, dos vivían en unión libre y uno era divorciado. En el segundo, había tres casados, tres en unión libre, una madre soltera, una divorciada y una viuda. Con respecto, al grupo III, se determinó que siete eran casados, una 
pareja se ubicó en unión libre y una viuda. En cuanto al tipo de familia, se observó que en el grupo I, ocho eran nucleares y dos extensas, tanto en el segundo y tercer grupo, la estructura familiar fue similar, cinco nucleares, dos extensas y dos monoparentales. La religión que predominó fue la católica en los tres grupos. En el aspecto socioeconómico, en el grupo I, siete familias se ubicaron en el nivel D, dos en el E y una en el C, con relación al segundo, se reportó que seis estaban en el D y tres en el E, en cuanto al grupo III, se detectó que ocho se localizaban en el D y una en el E. Lo anterior, difiere a lo reportado por Borges, et al. (2006), Manzano y Arranz (2008) quien señalaron que es más probable encontrar a niños con altas capacidades en familias con niveles socioeconómicos privilegiados, porque esta condición ayuda a que los padres les proporcionen recursos educativos adicionales a sus hijos que les proveen diversas oportunidades.

Otra de las preguntas indagó sobre cuáles personas de la familia ayudaban en las tareas escolares de los niños. Los datos revelaron que, en el primer grupo, se reportó en tres casos que la madre era quien asistía a sus hijos en las actividades, la diada de ambos padres se mencionó en dos ocasiones, el padre en una, la colaboración entre madre y hermanos mayores se indicó en otro de los entrevistados, también se señaló que tres de los niños hacían esta actividad de forma independiente. Con respecto al grupo II, en cinco de los niños se reportó que la madre era quien los apoyaba, a dos de los estudiantes los ayudaban el padre y los hermanos, en uno de los participantes fueron la madre y los hermanos; y en otro, la familia completa. En el tercer grupo, se reconoció que existe una mayor cantidad de miembros de la familia que colaboraron en dicha labor, de tal forma, se observó que las categorías de la madre, madre y hermanos; y, hermana fueron reportados en una ocasión respectivamente, en dos estudiantes se mencionó que ambos padres, otros dos indicaron que toda la familia y dos hacían la tarea solos (Tabla 5).

Tabla 5. Características de las familias por grupo de altas capacidades

\begin{tabular}{llll}
\hline & \multicolumn{1}{c}{ GRUPO I } & \multicolumn{1}{c}{ GRUPO II } & \multicolumn{1}{c}{ GRUPO III } \\
\hline Estado civil & - Siete casados & - Tres casados, & - Siete casados \\
& - Dos unión libre & - Tres unión libre & - Uno unión libre \\
& - Un divorciado & - Una madre soltera & - Una viuda \\
& & - Una divorciada & \\
& & - Una viuda & \\
\hline
\end{tabular}




\begin{tabular}{|c|c|c|c|}
\hline Tipo de familia & $\begin{array}{l}\text { - Ocho nucleares } \\
\text { - Dos extensas }\end{array}$ & $\begin{array}{l}\text { - Cinco nucleares } \\
\text { - Dos monoparentales } \\
\text { - Dos extensas }\end{array}$ & $\begin{array}{l}\text { - Cinco nucleares } \\
\text { - Dos monoparentales } \\
\text { - Dos extensas }\end{array}$ \\
\hline Religión & $\begin{array}{l}\text { - Nueve Católica } \\
\text { - Una no práctica }\end{array}$ & $\begin{array}{l}\text { - Ocho Católicas } \\
\text { - Una Cristiana }\end{array}$ & - Nueve Católicas \\
\hline $\begin{array}{l}\text { Nivel } \\
\text { socioeconómico }\end{array}$ & $\begin{array}{l}\text { - Una en el C } \\
\text { - Una en el D+ } \\
\text { - Seis en el D } \\
\text { - Dos en el E }\end{array}$ & $\begin{array}{l}\text { - Cuatro en el D+ } \\
\text { - Dos en el D } \\
\text { - Tres en el E }\end{array}$ & $\begin{array}{l}\text { - Cinco en el D+ } \\
\text { - Tres en el D } \\
\text { - Una en el E }\end{array}$ \\
\hline $\begin{array}{l}\text { Personas que ayudan } \\
\text { en las tareas }\end{array}$ & $\begin{array}{l}\text { - Tres la madre } \\
\text { - Uno el padre } \\
\text { - Dos ambos padres } \\
\text { - Uno la madre y hermanos } \\
\text { - Tres solos }\end{array}$ & $\begin{array}{l}\text { - Cinco la madre } \\
\text { - Uno la madre y los hermanos } \\
\text { - Dos el padre y los hermanos } \\
\text { - Uno toda la familia }\end{array}$ & $\begin{array}{l}\text { - Uno la madre } \\
\text { - Dos ambos padres } \\
\text { - Uno la madre y hermanos } \\
\text { - Uno hermana } \\
\text { - Dos toda la familia } \\
\text { - Dos solos }\end{array}$ \\
\hline
\end{tabular}

Información referente a las actividades escolares, extracurriculares y creencias de los padres con respecto a sus hijos

Se encontró que en el grupo I, siete niños realizaban alguna actividad extracurricular, en el segundo grupo, cinco estudiantes asistían a clases adicionales y en tercero, ocho alumnos acudían a otras disciplinas. Al respecto, los padres comentaron que les proporcionaron estas alternativas a sus hijos, porque continuamente les solicitaban desarrollar otras labores en sus tiempos libres, se debe mencionar que, una de las mamás explicó que a petición de su hija le agregaba tarea adicional para evitar que se aburriera.

Además, se preguntó si era común que recibieran quejas por parte del profesor en relación con la conducta de sus hijos, los datos revelaron que, en los tres grupos, los padres indicaron que el comportamiento de sus niños en el salón de clases era bueno y explicaron que cuando se les solicitaba que acudieran a la escuela, era para felicitarlos por el desempeño académico de los estudiantes. De igual manera, se observó que, en los tres grupos, los alumnos mantenían buenas relaciones con sus pares (Tabla 6). Al respecto, Gross (1999) señaló que los alumnos con altas capacidades en ocasiones en la escuela viven una relación escolar contradictoria, mientras sus logros académicos son valorados por los adultos y algunos compañeros, otros miembros de la comunidad escolar tienen una perspectiva distinta.

Es importante señalar que, todos los entrevistados indicaron estar de acuerdo con que sus hijos continúen sus estudios. También se cuestionó sobre hasta qué nivel escolar les gustaría 
que sus hijos estudiaran, la información reflejó que en el grupo I, a tres les agradaría que terminaran alguna licenciatura y siete que concluyeran un posgrado. En el segundo, se observó que tres de los padres desearían que realizaran una licenciatura y seis un posgrado. Con respecto al tercero, se detectó que dos progenitores aspiran a que sus hijos hagan una licenciatura y siete un posgrado. Posteriormente, se preguntó que de acuerdo con las características y habilidades que observaban en sus hijos hasta qué nivel creen que estudiarían, en el grupo I cinco padres respondieron que sus niños realizarán una licenciatura y otros cinco creen que lograrán algún posgrado. En el segundo, se encontró que uno terminaría la secundaria, dos la licenciatura y seis un posgrado. Los datos en el grupo III, mostraron que uno de los padres reportó que su hijo realizará una carrera técnica, uno licenciatura y siete piensan que estudiarán un posgrado (Tabla 6). Al respecto, Carreras (2010) señaló que cuando los padres se enteran de que sus hijos tienen altas capacidades incrementa el estrés familiar, ya que en ocasiones las expectativas de éxito vinculadas a las habilidades de los niños no son realistas, por lo es necesario brindarles orientación para que canalicen adecuadamente su potencial.

A los padres se les preguntó si contaban con los recursos económicos suficientes para apoyar los estudios de sus hijos, los resultados mostraron que en el grupo uno, cinco indicaron que sí y otros cinco que no. Los padres del segundo señalaron que cinco si podían apoyar a sus hijos económicamente en su preparación profesional, tres que no y uno no está seguro (Tabla 6). En cuanto a los participantes del grupo III, respondieron, seis que sí, uno que no y dos no están seguros. Lo anterior, reflejó que aun cuando a los padres les gustaría apoyar a sus hijos en los estudios, reconocen que sus posibilidades económicas en ocasiones no son suficientes, lo cual les genera miedo y ansiedad ante el futuro académico de los niños, tal y como lo refirió Carreras (2010).

Tabla 6. Datos reportados por los padres sobre el aspecto escolar y extraescolar

\begin{tabular}{|c|c|c|c|}
\hline & GRUPO I & GRUPO II & GRUPO III \\
\hline $\begin{array}{l}\text { Participa en otras actividades } \\
\text { extra escolares }\end{array}$ & $\begin{array}{l}\text { - Cuatro natación } \\
\text { - Uno música } \\
\text { - Uno futbol } \\
\text { - Uno gimnasia } \\
\text { - Tres ninguna }\end{array}$ & $\begin{array}{l}\text { - Dos gimnasia } \\
\text { - Uno Niño talento } \\
\text { - Uno música } \\
\text { - Uno banda de guerra } \\
\text { - Cuatro ninguna }\end{array}$ & $\begin{array}{l}\text { - Tres futbol } \\
\text { - Dos niño talento } \\
\text { - Uno baile } \\
\text { - Uno computación } \\
\text { - Uno gimnasia } \\
\text { - Uno ninguna }\end{array}$ \\
\hline
\end{tabular}




\begin{tabular}{|c|c|c|c|}
\hline & GRUPO I & GRUPO II & GRUPO III \\
\hline $\begin{array}{l}\text { Reciben quejas sobre la conducta } \\
\text { de su hijo }\end{array}$ & $\begin{array}{l}\text { - Uno si } \\
\text { - Ocho no } \\
\text { - Uno a veces }\end{array}$ & $\begin{array}{l}\text { - Ocho no } \\
\text { - Uno a veces }\end{array}$ & - Nueve no \\
\hline $\begin{array}{l}\text { Relaciones con sus compañeros } \\
\text { de clase }\end{array}$ & $\begin{array}{l}\text { - Nueve buena } \\
\text { - Uno regular }\end{array}$ & $\begin{array}{l}\text { - Siete buena } \\
\text { - Dos regular }\end{array}$ & $\begin{array}{l}\text { - Seis buena } \\
\text { - Tres regular }\end{array}$ \\
\hline $\begin{array}{l}\text { Nivel escolar que les gustaría } \\
\text { que estudiaran }\end{array}$ & $\begin{array}{l}\text { - Tres licenciatura } \\
\text { - Siete posgrado }\end{array}$ & $\begin{array}{l}\text { - Tres licenciatura } \\
\text { - Seis posgrado }\end{array}$ & $\begin{array}{l}\text { - Dos licenciatura } \\
\text { - Siete posgrado }\end{array}$ \\
\hline $\begin{array}{l}\text { Nivel escolar al que piensan que } \\
\text { lograran estudiar }\end{array}$ & $\begin{array}{l}\text { - Cinco licenciatura } \\
\text { - Cinco posgrado }\end{array}$ & $\begin{array}{l}\text { - Uno secundaria } \\
\text { - Dos licenciatura } \\
\text { - Seis posgrado }\end{array}$ & $\begin{array}{l}\text { - Una carrera técnica } \\
\text { - Una licenciatura } \\
\text { - Siete posgrado }\end{array}$ \\
\hline $\begin{array}{l}\text { Cuentan con los recursos } \\
\text { económico para apoyar sus } \\
\text { estudios }\end{array}$ & $\begin{array}{l}\text { - Cinco si } \\
\text { - Cinco no }\end{array}$ & $\begin{array}{l}\text { - Cinco si } \\
\text { - Tres no } \\
\text { - Uno no están seguros }\end{array}$ & $\begin{array}{l}\text { - Seis si } \\
\text { - Uno no } \\
\text { - Dos no están seguros }\end{array}$ \\
\hline
\end{tabular}

\section{Relación entre las variables personales y familiares}

Para conocer la relación entre las variables personales y familiares evaluadas en el estudio se empleó una correlación de Spearman Rho, los resultados revelaron que el tiempo que dedican las madres para realizar las tareas con sus hijos $\left(r_{s}=-.635^{* *}\right)$ y actividades extracurriculares $\left(r_{s}=-.384^{*}\right)$ se asocian de forma negativa con el autoconcepto académico. La nominación realizada por el docente se correlacionó de manera positiva con las actividades extracurriculares de los alumnos $\left(r_{s}=.383\right)$ y con la escolaridad de la madre $\left(r_{s}=.418\right)$. Con respecto, al nivel escolar de la madre, se encontró que esta favorece de forma positiva la búsqueda de actividades adicionales para la educación de sus hijos ( $\mathrm{r}=.389 *)$. En cuanto a los padres, se observaron correlaciones positivas y significativas entre la edad de ellos con la de las madres $\left(r_{s}=.729 * *\right)$, de igual forma la escolaridad de los padres y de las madres se asocia positivamente $\left(r_{s}=.559 * *\right)$. Fue interesante encontrar que, en la muestra estudiada, los resultados revelaron que el nivel socioeconómico de la familia se relacionó de forma negativa con actividades extracurriculares $\left(r_{s}=-.495^{* *}\right)$, con la escolaridad de la madre $\left(r_{s}=-.526^{* *}\right)$ y con el nivel educativo del padre $\left(r_{s}=-.512 * *\right)$.Ver Tabla 7 . 
Tabla 7. Correlación entre las variables personales y familiares

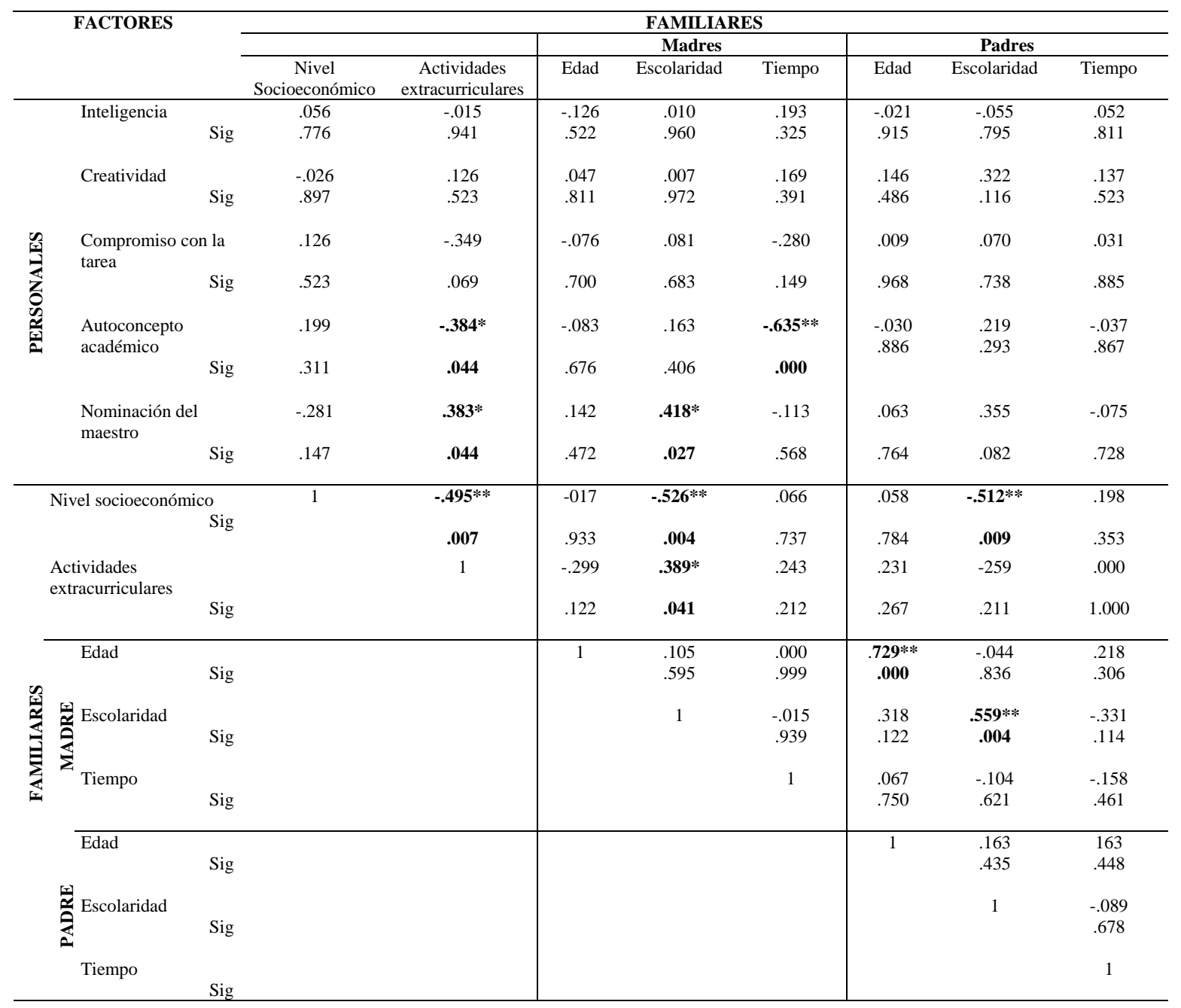

Discusión y Conclusiones

A partir de las variables evaluadas se encontró que un $4 \%$ de los niños presentó altas capacidades, estos datos concuerdan con lo reportado en otros estudios en los cuales se observó una prevalencia de un 3\% a un $20 \%$ de estudiantes de primaria ubicados en esta categoría (Armenta, 2008; Cervantes et al., 2011; Chávez et al., 2009; Espinoza \& Reyes, 2008). Además, se encontró que esta población pertenece a un grupo heterogéneo, con distintos tipos de habilidades y talentos, de ahí la necesidad de incluir diversas variables para lograr una 
identificación adecuada y conocer sus capacidades (Armenta, 2008; Gentry \& Knight, 2011; Manzano, Arranz \& Sánchez de Miguel, 2010; Ordaz \& Acle, 2013).

Se encontró que 12 de los niños detectados eran primogénitos, seis nacieron en segundo lugar, ocho en el tercero y dos en cuarto. Estos datos permiten reflexionar sobre lo reportado por Kristensen y Bjerkedal (2007), Ordaz y Acle (2013) Zacatelco y Acle (2009), al reconocer que es más común encontrar características de altas capacidades en los primogénitos, la explicación para esto es que los padres les brindan más tiempo, atención y otras oportunidades para desarrollar sus capacidades a los hijos mayores.

En cuanto al aspecto emocional, 19 (68\%) de los alumnos con altas capacidades fueron reportados como sensibles, cinco como amables, tres tímidos y uno hiperactivo. Para Campo (2016) los niños ubicados en esta categoría tienden a ser pasivos socialmente e inhibir sus emociones, lo cual los ubica en riesgo social y vulnerabilidad, de ahí la importancia de realizar programas para favorecer sus habilidades de interacción con sus pares.

En cuanto al análisis de la influencia de los factores de la madre se encontraron datos interesantes, por ejemplo, el nivel mínimo de estudio fue la secundaria y el máximo la licenciatura. Se observó que, 18 (64\%) de ellas se dedicaban al hogar y al cuidado de sus hijos. Aun cuando algunas indicaron que trabajaban, se reportó que 25 (83\%) empleaban tiempo para realizar la tarea con sus hijos y en ocasiones otros miembros de la familia las apoyan en esta actividad. Lo anterior, permite reconocer que el tiempo dedicado por las madres a sus hijos en relación con las tareas escolares favorece el desarrollo de las capacidades cognitivas (Manzano \& Arranz, 2008). En el caso de los padres, se reportó como nivel educativo mínimo la primaria y máximo la maestría, sus ocupaciones fueron heterogéneas y aunque algunos reportaron que estas labores representaban largas jornadas, $15(54 \%)$ de ellos dedicaban un espacio para hacer la tarea con sus hijos. Al respecto, Pougnet, et al. (2011) mencionaron que la implicación paterna en la educación de los niños favorece el desarrollo adecuado de la esfera cognitiva y emocional. 
También, se reportó que $24(86 \%)$ de los padres eran casados o vivían en unión. Lo anterior, demuestra que los niños se crecen dentro de un núcleo familiar que cubre sus necesidades básicas, en donde sus miembros se involucran en las actividades escolares. Estos resultados revelaron que el apoyo familiar, el grado de acompañamiento de los padres y el tiempo que dedicaron a sus hijos, representaron elementos relevantes en el desarrollo de las habilidades y en el proceso educativo de los estudiantes. Al respecto, Bazán, Sánchez y Castañeda (2007) indicaron que cuando los padres proporcionan tiempo y dedicación al aprendizaje de los niños se logra un mejor desempeño académico.

Otro elemento interesante fue que las familias de los alumnos con altas capacidades en este estudio pertenecen a los estratos más bajos de acuerdo con el AMAI, a pesar de esto, se observó que existe un apoyo familiar importante de todos los miembros en la educación de los niños y que independientemente del nivel socioeconómico 20 (71\%) de los estudiantes realizaban actividades extracurriculares. Lo que permite reconocer la existencia de alumnos con altas capacidades que habitan en zonas de marginación social, con condiciones precarias, en ambientes de pobreza lo que pueden afectar su rendimiento escolar y emocional, pero si la familia les brinda el soporte necesario sus capacidades se favorecen (Acle \& Ordaz, 2010; Burley, Barnard-Brak, Marbley \& Deason, 2010; Rodríguez \& Valdivieso, 2008). Lo anterior, es de suma importancia dado que los resultados contradicen a los reportados por Borges, et al. (2006), Manzano y Arranz (2008) en los cuales se encontró que es más probable encontrar a niños con altas capacidades en familias con niveles socioeconómicos privilegiados, porque esta condición ayuda a proporcionarles recursos educativos adicionales que les provee de otras oportunidades. En este sentido, Acle y Ordaz (2010), Rodríguez y Valdivieso (2008), señalaron que el pertenecer a un estatus económico elevado no es el único aspecto asociado a los alumnos de esta categoría. De ahí que, la hipótesis referente a que existen distintas variables familiares que actúan como moderadoras de las altas capacidades se apoya con los resultados encontrados en esta investigación.

Por otro lado, se reportó que $25(89 \%)$ de los estudiantes tenían buena conducta y 27 (96\%) se llevaban bien con sus compañeros de clase. Dichos resultados coinciden con los 
observados por Pfeiffer (2008) quien reconoció que los niños con altas capacidades suelen demostrar sensibilidad hacia las necesidades de los demás y disfrutan de las relaciones sociales. Por lo que el desarrollo de estas características depende en gran medida a los vínculos y la forma en que los individuos son educados dentro de su contexto familiar y escolar.

Un hallazgo interesante, fue que los padres de los niños detectados mostraron altas expectativas con respecto al nivel de escolaridad que pueden alcanzar sus hijos en un futuro, pero son conscientes de que sus recursos económicos son bajos y que tendrán que esforzarse para apoyarlos. Esto se relacionó con lo reportado por, Borges et al. (2006), Gómez y Valadez (2010) al señalar que los padres demuestran sentimientos de satisfacción y en ocasiones temor por no contar con la solvencia necesaria para ayudar a sus hijos en sus estudios. En este sentido, Kitano (2003) reconoció la importancia de que el sistema educativo y el gobierno brinden becas económicas a los estudiantes con altas capacidades que se encuentran en desventaja social.

En cuanto a las asociaciones entre los factores personales y familiares, se determinó que los docentes nominan a los niños que realizan más actividades extracurriculares, a su vez dicha variable ayuda a que los alumnos reconozcan sus destrezas y habilidades por tanto son autocríticos de sus propias capacidades. Un dato interesante fue que a pesar de que los padres de familia tienen un nivel socioeconómico bajo, ellos buscan alternativas adicionales para favorecer las habilidades de sus hijos. Lo anterior indicó que aun cuando su condición económica es precaria, ellos se involucran en la educación de sus hijos y les brindan alternativas adicionales para favorecer sus capacidades. Por otro lado, se encontró que, la escolaridad de las madres se vinculó con la cantidad de actividades extracurriculares que realizan los niños y con la nominación del maestro, lo que refleja que la percepción del docente es distinta cuando reconocen que los padres tienen un mayor nivel de estudios. En este sentido, los hallazgos son similares a lo reportado por Manzano y Arranz (2008), Nepper y Beauregard (2011) quienes señalaron que el nivel cultural materno y el hecho de dedicarse al hogar promueven las habilidades de los estudiantes, porque están directamente a cargo del cuidado de sus hijos y examinan alternativas que a ellos les gusten. 
El tiempo que dedican las madres en hacer las tareas escolares y la cantidad de actividades extraescolares se relaciona de forma negativa con la autopercepción académica de los niños. Lo anterior, se puede deber a que si existen altos niveles de tensión y estrés en el niño estos afectan su forma de valorar sus propias capacidades (Manzano \& Arranz, 2008; Sękoski \& Siekańska, 2008). Asimismo, Garn et al. (2010) indicaron que las actitudes y comentarios de los padres influyen en la motivación y el autoconcepto de los alumnos, porque son variables que se regulan a través de la interacción social.

También, se encontró que la escolaridad de las madres y los padres no determina el nivel socioeconómico. Lo anterior, es de particular interés porque pone de manifiesto que existe una desigualdad entre el nivel educativo y el ingreso percibido, tal y como lo describió Valdivia y Pedrero (2011), al indicar que aun cuando el sueldo promedió ha aumentado, la mayoría de los trabajadores experimentan un detrimento importante en sus percepciones. Pese a esta condición, los padres se mostraron comprometidos con la educación de sus hijos y les proporcionan actividades extracurriculares de acuerdo con sus posibilidades.

Se concluye que la evidencia empírica de este estudio resalta el papel trascendente que tienen los padres en el desarrollo de los talentos de sus hijos con altas capacidades, de ahí la importancia de brindarles los apoyos necesarios para que les provean de las estrategias educativas acordes para favorecer sus habilidades, además es indispensable el trabajo colaborativo entre el docente y los padres para lograr un óptimo conocimiento de los estudiantes.

\section{Referencias}

Acle, T. G. \& Ordaz, V. G. (2010). Resiliencia y aptitudes sobresalientes en niños de zonas urbano-marginadas. Ideacción, 31, 288-299

Armenta, O. C. (2008). Educación incluyente para sobresalientes en las sociedades del conocimiento. Revista Internacional de Ciencias Sociales y Humanidades, 1, 109-131. 
Asociación Mexicana de Agencias de Mercados y Opinión Pública. (2017). Institución de investigaciones sociales S.C. Recuperado AMAI http://www.fergut.com/clasificacion-deniveles-socioeconomicos-en-mexico-segun-la-amai

Bazán, R. A., Sánchez, H. B. A. \& Castañeda, F. S. (2007). Relación estructural entre apoyo familia, nivel educativo de los padres, características del maestro y desempeño en la lengua escrita. Revista Mexicana de Investigación

Bernal, A., Esparza, J., Ruíz, M. J., Ferrando, M. \& Sainz, M. (2017). Especificidad de la Creatividad: Figurativa y Científica. Electronic Journal of Research in Educational Psychology, 15 (3), 574-597.

Blanco, V. M. C. (2001). Guía para la identificación y seguimiento de alumnos superdotados. España: CISS Praxis.

Borges, R. A., Hernández, J. C. \& Rodríguez, N. E. (2006). Comportamientos parentales en familias con superdotados. Faísca, 11(13), 48-58.

Burley, H., Barnard-Brak, L., Marbley, F. A. \& Deason, C. (2010). African american millennials a profile of promise. Gifted Child Today, Spring 32(2), 47-54.

Campo, R. M. (2016). Regulación emocional y habilidades sociales en niños con altas capacidades intelectuales. (Tesis de Doctorado Inédita), Universidad Complutense de Madrid, España.

Carreras, T. L. (2010) Repercusiones emocionales del niño/a con altas capacidades intelectuales. Recuperado de http://www.mentor.cat/uploads/2/5/2/4/25246316/repercusiones.pdf

Catellanos, S. D. Bazán, R. A. Ferrari, B. A. M. \& Hernández, R. C. A. (2015) Apoyo familiar en escolares con alta capacidad intelectual de diferentes contextos socieducativos. Revista de Psicología, 33 (2). 299-332.

Cervantes, D., Valadez, M. D., Lara, B., Zambrano, G., Pérez, L., López, E. \& Avelino, I. (2011). Identificación de alumnos con talento en una escuela Secundaria de la Zona Metropolitana de Guadalajara. Revista de educación y Desarrollo, 18, 41-50.

Chamberlin, M. \& Chamberlin, S. (2010). Enhancing preservice teacher development: Field Experiences with Gifted Students. Journal for the Education of the Gifted, 33(3), 381-416.

Chan, D. (2005). Family environment and talent development of chinese gifted students in Hong Kong. Gifted Child Quarterly, 4(3), 211-221. 
Chávez, S. B. (2014). Evaluación multidimensional de alumnos con aptitudes sobresaliente de Educación primaria. (Tesis de Doctorado Inédita), Facultad de Estudios Superiores Zaragoza, Universidad Nacional Autónoma de México, México.

Chávez, S. B., Zacatelco, R. F. J. \& Acle, T. G. (2009). Programa de enriquecimiento de la creatividad para alumnas sobresalientes de zonas marginadas. Electronic Journal of Research in Educational Psychology, 15 (3), 533-552

Costley, K. (2011). Descriptions of a quality gifted school and recommendations to parens today. Recuperado de http://eric.ed.gov/?q=what+does+it+ mean+to+say+a+school+is+doing+well +Eisner\&ft=on\&ff1=dtySince_1994\&ff2=subEducational+Change\&pg=11\&id=ED519930

Elices, S. J. A., Palazuelo, M. M., \& Del Caño, S. M. (2006). El profesor identificador de necesidades educativas asociadas a las altas capacidades. Faisca, 11(13), 23-47.

Endepolhs-Ulpe, M. \& Ruf, H. (2005). Primary School Teacher's Criteria for the Identification of Gifted Pupils. High Ability Studies, 15(2), 219-228.

Espinoza, V. M. \& Reyes F. P. M. (2008). Principales características y necesidades psicopedadógicas del alumnado con altas capacidades en un centro de educación primaria. Aula abierta, 36(1),49-64.

Ferrando, M., Prieto, M. D. Ferrándiz, C. \& Sánchez, C. (2005). Inteligencia y creatividad. Revista Electrónica de Investigación Psicoeducativa, 7(3), 21-50.

Gagné. F. (2012). Construyendo el talento a partir de la dotación: breve revisión del MDDT 2.0. En Valadez, S. M. D., Betancourt, M. J. \& Zavala B. M. A. (Eds.), Alumnos Superdotados y Talentosos. Identificación, Evaluación e Intervención Una Perspectiva para Docentes (pp. 45-54). México: Manual Moderno.

Garn, A., Matthews, M. \& Jolly, J. (2010). Parental influences on the academic motivation of gifted students: A self-determination theory perspective. Gifted Child Quarterly, 54(4), 263272.

Gentry, R. \& Knight, T. (Abril, 2011). Simply Gifted: Their Attributes through the Eyes of College Students. Ponencia Presentada en The 2011 International conference "Peace through Understanding”, Jackson, Mississippi, Estados Unidos.

Gómez, P. M. A. \& Valadez, S. D. (2010). Relaciones de la familia y del hijo/a con superdotación intelectual. Faísca, 15(17), 67-85. 
Gross, M. U. (1999). Exceptionally Gifted Children. New York: Routledge.

Hernández, J. C. \& Borges, R. A. (2010). Entorno escolar del alumnado de altas capacidades intelectuales frente a sus compañeros de distinto nivel de inteligencia. Faísca, 15(17), 36-49.

INEGI (2015). Anuario Estadístico y Geográfico del Distrito Federal. Recuperado de http://internet.contenidos.inegi.org.mx/contenidos/productos/prod_serv/contenidos/espanol/b vinegi/productos/nueva_estruc/anuarios_2015/702825076924.pdf

Kerlinger, F. \& Lee, H. (2001). Investigación del Comportamiento. Métodos de Investigación en Ciencias Sociales. México: McGraw Hill.

Kitano, M. (2003). Gifted potential and poverty: A call for extraordinary action. Journal for the Education of the Gifted, 26(4), 192-303.

Kristensen, P. \& Bjerkedal, T. (2007). Explaining the relation between birth order and inteligence. Science, 316, 1717-1725.

los adolescentes talentosos. Faisca, 15(17), 2-17.

Manzano, A. \& Arranz, E. B. (2008). Contexto familiar, superdotación, talento y altas capacidades. Anuario de Psicología, 39(9), 289-309.

Manzano, A., Arranz, E. \& Sánchez de Miguel, M. (2010). Multi-criteria Identification of Gifted Children in a Spanish Sample. European Journal of Education and Psychology, 3(1), 5-17.

Martín, L. M. P. (2004). Niños inteligentes. España: Palabra.

Mönks., F. \& Van Boxtel, H. (1992). General, social and academic self-concepts of gifted adolescents. Journal of Youth and Adolescents, 21(2), 169-186.

Morawska, A. \& Sanders, M. (2009). Parenting gifted and talented children: conceptual and empirical foundations. Gifted Child Quarterly, 53(3),163-173.

Nepper, F. J. \& Beauregard, E. (2011). Longitudinal change and maternal influence on occupational aspirations of gifted female American and German

Ocampo, N. M. G. \& López, A. G. (2011). Inteligencia emocional en alumnos con alta capacidad y/o aptitudes sobresalientes en Iguala, Guerrero. Revista Mexicana de Psicología. Número especial, 992- 994.

Olszewski-Kubilius, P. (2008). The role of the family in talent development. En S.I. Pfeiffer (Ed.), Handbook of giftedness in children: Psycho-educational theory, research, and best practices (pp. 53-70). New York, NY: Springer. 
Ordaz, V. G. \& Acle, T. G. (2013). Perfil psicosocial de adolescentes con Aptitud Sobresaliente de Bachillerato Público. Electronic Journal of Research in Educational Psychology, 10(3), 1267-1298.

Pérez, S. L. \& González, B. C. (2007). Diferencias evolutivas y variabilidad intelectual entre estudiantes con alta capacidad. Faísca, 12 (14), 17-29.

Piechowski, M., y Colangelo, N. (1984). “Developmental Potential of the Gifted”. Gifted Child Quarterly, 28: 80-88

Piirto, J. \& Fraas, J. (2012). A mixed-methods comparison of vocational and identified gifted high school students on the Overexcitability Questionnaire. Journal for the Education of the Gifted, 35(1), 3-34.

Piirto, J. (1999). Talented children and adults: Their development and education. E. U: Prentice Hall/Merrill.

Pougnet, E. Serbin, L. Stack, D. M. \& Schwartzman, A.E. (2011). Fathers' Influence on Children's Cognitive and Behavioural Funtioning: A Longitudinal Study of Canadian Families. Canadian Journal of BehaviouralScience. 4(3), 173-182.

Preckel, F., Goetz, T., Pekrun, R. \& Kleine, M. (2008). Gender differences in gifted and averageability students. Gifted Child Quarterly, 52(2),146-159.

Prieto, D. (1999). Identificación, Evaluación y Atención a la Diversidad del Superdotado. España: Aljibe.

Raven, J. C., Court, J. H. \& Raven, J. (1993). Test de Matrices Progresivas Raven. Escala Coloreada, General y Avanzada. Manual. Buenos Aires: Paidós.

Renzulli, S. J. (1986). Systems and models for developing programs for the gifted and talented. USA: Creative Learning Press.

Renzulli, S. J. (2011). What Makes Giftedness? Reexamining a Definition. Kappan, 92(8), 81-89.

Rodríguez, S. C. R. \& Valdivieso, M. A. G. (2008). El éxito escolar de alumnos en condiciones adversas. Revista Lationamericana de Estudios Educativos. XXXVIII, (12), 81-106.

Rudasill, M., Capper, C., Foust, Callahan, M. \& Albaugh, S. (2009). Grade and gender differences in gifted students' self-concepts. Journal for the Education of the Gifted, 32(3), $340-367$. 
Sarouphim, K. (1999). Discovering multiple intelligence through a performance-based assessment: Consistency with independent ratings. Exceptional Children, 65, 151-16.

Schick, H. \& Phillipson, S. N. (2009). Learning Motivation and Performance Excellence in Adolescents with High Intellectual Potential: What Really Matters? High Ability Studies, 20(1),15-37.

Sękoski, A. \& SiekSańska, M. (2008). National academic award winners over tine: their family situation, education and interpersonal relations. High Ability Studies, 19(2), 155-171.

Torrance, P. (2008). Research Review for the Torrance test of Creative Thinking Figural and Verbal Forms $A$ and $B$. USA: Scholastic Testing Service. Inc.

Valdés, C. A. A., Sánchez, E. P. A. \& Yañéz, Q. A. I. (2013). Perfiles de estudiantes mexicanos con aptitudes intelectuales sobresalientes. Acta Colombiana de Psicología, 16(1), 25-33.

Valdivia, L. M. \& Pedrero, N. M. (2011). Segmentación Laboral, Educación y Desigualdad Salarial en México. Revista Mexicana de Sociología, 73(1), 139-175.

Zacatelco R. F. J., Chávez, S. B. \& González, G. A. (Julio, 2013). Análisis Psicométrico de una Escala de Nominación del Maestro para Identificar Aptitudes Sobresalientes: Resultados preliminares. Presentado en el XXXIV Congreso Interamericano de Psicología "Por La Integración De Las Américas”, Brasilia, Brasil.

Zacatelco, R. F. J. \& Acle, T. G. (2009). Validación de un modelo de identificación de la aptitud sobresaliente en estudiantes de primaria. Revista Mexicana de Investigación en Psicología,1(1), 49-53.

Zacatelco, R. F. J. \& Chávez, S. B. (2010). Alumnos Sobresalientes: identificación y Diseño de Programas en Escenarios de Educación Primaria. Ideacción, Edición especial, 300-309.

Zacatelco, R. F. J. (2005). Modelo para la Identificación del niño sobresaliente en escuelas de educación primaria. (Tesis de Doctorado Inédita), Facultad de Estudios Superiores Zaragoza, Universidad Nacional Autónoma de México, México.

Zeynep, I. H. \& Bayindir, N. (2009). Awareness Level of Teacher About the Characteristics of Gifted Children. Australian Journal of Basic and Applied Sciences, 3(3), 2519-2527. 